\title{
Research on the Flatness Measuring Technology of Satellite Antennas under Thermal Loads
}

\author{
Mao Liang \\ Engineering Systems Division \\ The 38th Research Institute of CETC \\ Hefei, China \\ Maoliangcetc38@163.com
}

\author{
Wang Mei \\ Engineering Systems Division \\ The 38th Research Institute of CETC \\ Hefei, China \\ wangmei@cetc38.com
}

\begin{abstract}
This paper has done some research on the flatness measuring method of satellite antennas under thermal loads. A real time thermal control technique was proposed. Using this technique, the temperature of the satellite antenna was heated from the room temperature to $70^{\circ} \mathrm{C}$, and the temperature distribution of the antenna was wellproportioned and stable. Based on the photogrammetry, a flatness measuring method of satellite antennas under thermal loads was proposed. The full-field displacement of the antenna was approved. This paper provided a new technology for the flatness measuring of satellite antennas under thermal loads, and applied it on a satellite antenna sub array. The measuring results provided references for structure design and optimization of satellite antennas.
\end{abstract}

Keywords-satellite antennas; flatness measuring; temperature controlling; photogrammetry; binocular-vision

\section{INTRODUCTION}

Antennas are the most important part of satellite radars. As the demand of antenna resolution raises up, the antenna aperture is growing larger and larger and the flatness precision requirement of the antenna reflecting surface is becoming higher and higher. For phased array antennas, which are comprised by planar arrays, the flatness precision of its planar antenna has directly effect on indexes of its electromagnetic performance, such as antennas' gain, minor lobe, beam pointing accuracy, and so on. Therefore, the measurement of the flatness precision is getting more and more attention. The traditional measuring method is three-coordinate measuring system, which mostly works on straight guide rail motion measurement. Thus it is hardly applied on measurement in antenna fabrication or installation. In recent years, many three-dimension precision measuring equipment have been developed, as the relevant technology keeping on improving. Such as dual electronic transit theodolite industrial measuring system [1-2], laser tracking interferometer system [3], etc. Application of these measuring systems is limited by their precision and strict demand of the measuring environment. Hence, they are not capable of high-precision full-field measurement, especially under thermal loads, which are exactly requirements of flatness precision measurement. On the other hand, photogrammetric system [4] progress very fast in last twenty years. Being an optical measuring method, the high precise made it an excellent industrial measurement [5-8]. But it was difficult to find any reports about its application on temperature environment. This paper has brought photogrammetric system in measuring flatness precision of satellite antennas, which was under thermal loads.

\section{FLATNESS PRECISION REQUIREMENT OF SATELLITE ANTENNAS UNDER THERMAL LOADS}

The space environment of satellites is very complicate [9]. Temperature is one of the most important environmental factors [10]. Influenced by multiple factors, such as vacuum in space, radiation from sun and earth, etc., the working temperature of satellite antennas spans a very large range. To ensure a reliable working status for the measuring equipments on satellite antennas, the antennas' temperature should be controlled with high degree of accuracy. Hence, a temperature control device with high uniformity and stability are badly in need of. With this temperature control technology, the flatness of a satellite antenna sub array was measured in use of photogrammetry.

\section{TEMPERATURE CONTROL TECHNOLOGY}

To measure the flatness of satellite antennas under thermal loads, the key technology is accurately control the temperature. Traditional heating methods, such as black body and heating platform, cannot raise the antenna temperature uniformity and stably, because antennas usually have large area and complex inside structure.

\section{A. Temperature Control Device}

In this experiment, electrical heating belts, connected with one TN99D high-precision temperature controller, were introduced in to heat the antenna sub-array. The thermocouple was placed on the sub-array to detect its temperature, and real-time feedback the temperature information to the temperature controller. In this case, the accuracy of adjustment can reach to $0.1^{\circ} \mathrm{C}$. For the structure of the antenna sub-array being complex, its temperature adjustment accuracy was relied on the distribution of electrical heating belts. Applying this device on a satellite slotted waveguide antenna sub-array, the device and the distribution of electrical heating belts were 
shown in Fig .1. In order to uniformity raise the sub-array temperature, electrical heating belts were arranged on and around the sub-array in some regular pattern. During the adjustment phase, a FLIR infrared thermal imager was used to real-timely monitoring the temperature distribution of the sub-array. When the antenna temperature altered, the amount of its reflected infrared energy changed. The infrared thermal imager detected those energy change, and translated it into electrical signal by photoelectric sensor, and shown these signal in the form of gray scale or pseudo-color images, which shown the full-field temperature distribution. The minimum detectable temperature change was $0.01^{\circ} \mathrm{C}$. Based on the real-time feedback of the infrared thermal imager, the arrangement of the electrical heating belts was adjusted timely. Finally the antenna sub-array temperature can be raised up uniformly.

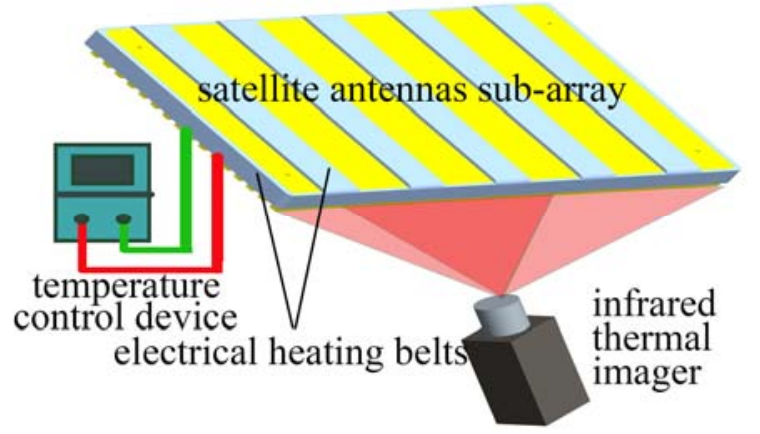

Figure 1. Temperature control device and the electrical heating belts arrangement.

\section{B. Uniformity and Stability Analysis of the Temperature Control Device}

To test the uniformity of the temperature control device mentioned above, the antenna sub-array temperature was successively raised to $30^{\circ} \mathrm{C}, 40^{\circ} \mathrm{C}, 50^{\circ} \mathrm{C}, 60^{\circ} \mathrm{C}$ and $70^{\circ} \mathrm{C}$. And at least 40 minutes were kept at these five temperatures. By the infrared thermal imager, the uniformity and stability of the temperature distribution on the antenna sub-array were monitored. In the monitored area of the infrared thermal imager, five typical points were chosen and shown in Fig .2. Temperature variations of these points were monitored in a series of the heating up and heat preservation progress. Temperature data were collected and reflected the uniformity and stability of the full monitoring area.

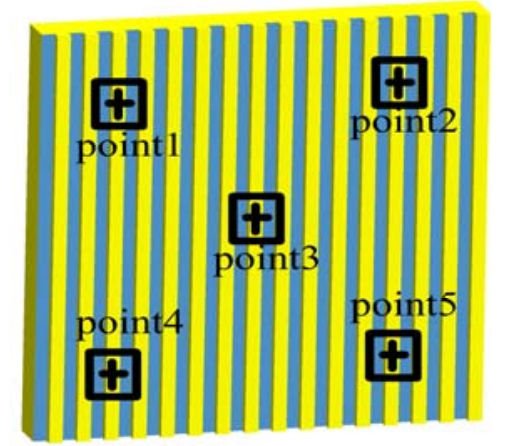

Figure 2. The distribution of the five monitoring points.

Sample frequency of the infrared thermal imager is 10 Hz. Its monitoring results were shown in Fig .3. The horizontal axis was the collecting time and the vertical coordinates was temperature of the monitoring point. Five curves were fitted by the temperature at corresponding time and able to show the temperature variation of the five points during the entire time history. Through the experiment data, it was observed that the five curves were highly superposition, which illustrated that temperature of the five positions were very close at the same time and proved that the temperature variation of the antenna subarray was highly uniformly. Some key temperature data of the curves at the five stable temperature $\left(30^{\circ} \mathrm{C}, 40^{\circ} \mathrm{C}, 50^{\circ} \mathrm{C}\right.$, $60^{\circ}$ Cand $70^{\circ} \mathrm{C}$ ) were listed in Tab .1. In this table, the maximum value was the biggest value of the five curves at a certain stable temperature; the minimum value was the corresponding smallest value, and the temperature fluctuation was the corresponding absolute value of the differential between the maximum and the minimum values. Data shown that temperature can be kept stable at least 40 minutes. And the temperature fluctuation were able to be controlled in $1.5^{\circ} \mathrm{C}$ respectively at $30^{\circ} \mathrm{C}, 40^{\circ} \mathrm{C}$, $50^{\circ} \mathrm{C}$ and $60^{\circ} \mathrm{C}$, while $2^{\circ} \mathrm{C}$ at $70^{\circ} \mathrm{C}$. It was proved that the temperature control device was enormously stable. Based on the satellite antennas environment test requirement in GJB 1027-90, the temperature uniformity and stability demanding are extremely high. And the analysis above show that this temperature control technology satisfied the strict demands.

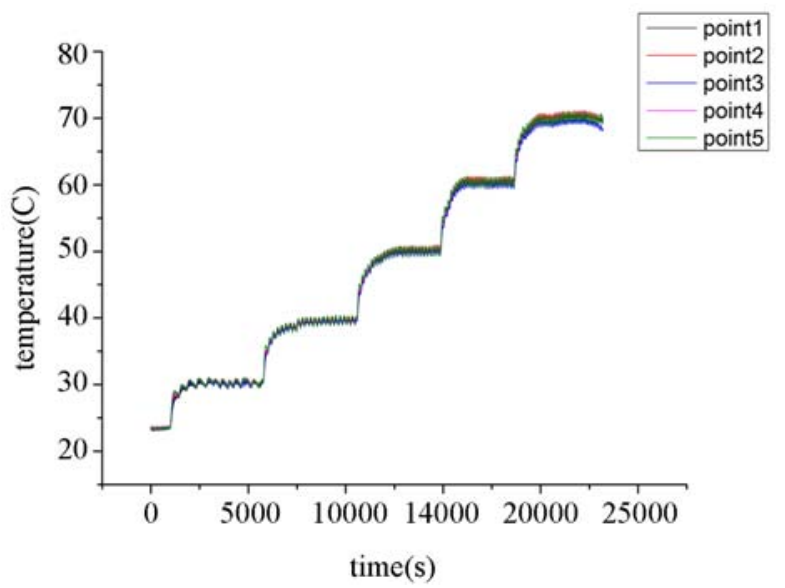

Figure 3. Temperature variation curves of five monitoring points.

TABLE I. Key TEMPERTURE DATA OF THE CURVES IN Fig .3.

\begin{tabular}{|l|c|c|c|c|c|}
\hline & $\mathbf{3 0}^{\circ} \mathbf{C}$ & $\mathbf{4 0}^{\circ} \mathbf{C}$ & $\mathbf{5 0}^{\circ} \mathbf{C}$ & $\mathbf{6 0}^{\circ} \mathbf{C}$ & $\mathbf{7 0}^{\circ} \mathbf{C}$ \\
\hline Maximun value $/{ }^{\circ} \mathbf{C}$ & 30.42 & 40.38 & 50.75 & 61.02 & 71.32 \\
\hline Minimun value $/{ }^{\circ} \mathbf{C}$ & 29.45 & 39.49 & 49.62 & 59.56 & 69.54 \\
\hline Fluctuation $/{ }^{\circ} \mathbf{C}$ & 0.97 & 0.89 & 1.13 & 1.46 & 1.78 \\
\hline
\end{tabular}

IV. Flatness Measuring of Satellite Antennas UNDER THERMAL LOADS

\section{A. Experimental Scheme}

Selection of displacement measurement is depended on the specific feature and working condition of the measuring object. There are many common industrial measuring methods used for measuring displacement, such as displacement sensors, photo-electricity position detectors (PSD) laser measurement and photogrammetry. Displacement sensors are unfit for measurement under 
thermal load, in consideration of their working temperature, mostly room temperature, and direct contacting measuring pattern. Dispensing with direct contaction, PSD laser measurement can overcome the problem caused by thermal load. But it is only able to detect single point, which is obviously unfit for measuring objects with large array and more than one interested places. Overcoming shortcomings of the two measuring methods mentioned before, photogrammetry can obtain the whole-field displacement of the interested surface. And it is also a noncontact measurement.

A photogrammetric system collects image data mainly through monocular-vision principle, binocular-vision principle and multi-vision principle. In this paper, the measuring system is based on the binocular vision principle. It is comprised by a couple of high precision real-time measuring cameras, controller and system software, as shown in Fig .4. In charged by the controller, two or more real-time photos of the measuring area are collected. Playing the role of a core processor, system software obtained three dimensional coordinates of feature points on the sub array, by processing the real-time photos.

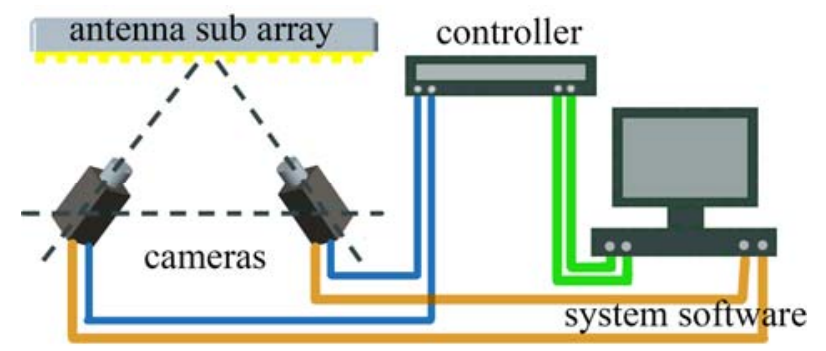

Figure 4. The schematic diagram of a photogrammetric system.

The detail measurement procedure was declared as follow:

- First, making feature points on satellite antenna sub-array surface. Feature points, carrying information of the measuring object's morphology changes, usually employing the object's own texture, can hardly be recognized from the surface of satellite antenna sub array, because this surface is processed to be very smooth without any macroscopic texture. Thus, we stamped the surface of the sub array by manually painting ink points on it. In this way, ink points played the role of feature points. And, without any contacting movement in usual displacement sensor detecting, the sub-array kept exactly the original status after the measurement.

- Second, employing a torque spanner, the sub-array was fixed on supports using certainly torque. The boundary conditions setting of the sub-array were shown in Fig .5.

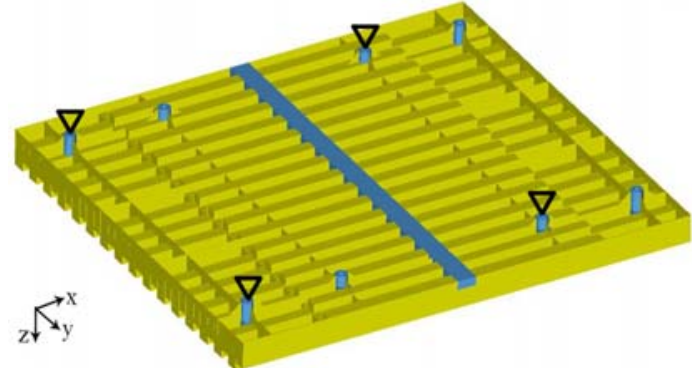

Figure 5. The boundary conditions setting of the sub-array. The black triangle marked positions of the clamped screw holes.

- Third, a couple of high precision real-time measuring cameras were employed as major detectors, field of which were coincident on the sub-array. The field of this measuring system was $3 \mathrm{w} / 4 \times 3 \mathrm{~h} / 4$, while the sub-array size was $w \times h$.

- Forth, heated by the temperature control device, temperature of the sub-array raised respectively from the room temperature to $30^{\circ} \mathrm{C}, 40^{\circ} \mathrm{C}, 50^{\circ} \mathrm{C}$, $60^{\circ} \mathrm{C}$ and $70^{\circ} \mathrm{C}$. More than two images of the subarray were captured by the controller at every temperature.

- Fifth, with regard to every stable temperature status, three-dimension coordinates of feature points were calculated by the system software, employing the digital speckle correlation method [11]. Thus the full-field displacement could be deduced.

\section{B. Experiment Results}

As shown in Fig. 6, the out-plane-displacement cloud picture of the antenna sub array surface was calculated by the system software. In this picture, areas with color closed to the top of the color code (at the right of the picture) have larger displacements. And oppositely, areas with color closed to the bottom of the color code have smaller displacements. The results shown as follow:

- First, the antenna sub-array deformed under the thermal load. The absolute value between the maximum and minimum values of the out-planedisplacement was lower than permissible value.

- Second, the position of clamped screw holes were $\mathrm{x}$ axial symmetry. And the out-plane-displacement in the cloud picture were also $\mathrm{x}$ axial symmetry, which was accord with the clamping pattern.

- Third, there were many slits on the satellite slotted waveguide antenna surface. Feature points cannot be fabricated on the slotted place. Therefore, the measuring result in Fig .6 was comprised by many independent small measureable areas.. 


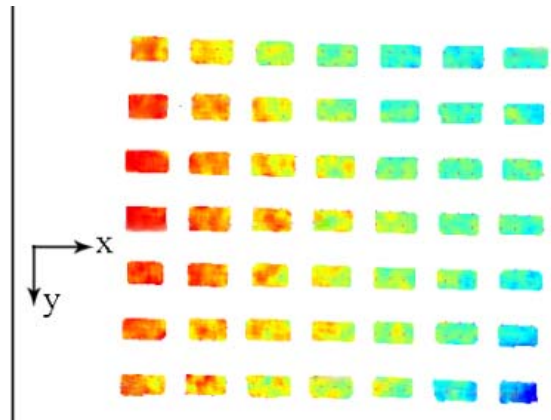

Figure 6. The out-plane-displacement cloud picture of the antenna sub-array surface.

\section{CONCLUSION}

To measure the flatness method of satellite antennas under thermal loads, firstly, this paper presented a real time thermal control technique. The antenna sub-array was heated by electrical heating belts, connected with a TN99D high-precision temperature controller. The temperature of the sub-array was monitored by a FLIR infrared thermal imager in real time. When the temperature of the satellite antenna sub-array was heated from the room temperature to $70^{\circ} \mathrm{C}$, the temperature displacement of the antenna was uniform and stable. And the temperature fluctuation can be controlled in $2^{\circ} \mathrm{C}$ within 40 minutes, which was satisfied the experiment demand. Secondly, based on photogrammetry, a flatness measuring method of satellite antennas under thermal loads was proposed. And the fullfield displacement of a satellite antenna sub array was approved. This method has advantages in whole-field and noncontact measuring, which is of great significance in satellite antennas measurement.

\section{REFERENCES}

[1] Quan Zhiming, "Study on Techniques of Precision Measurement in Assembly of Spacecraft Antenna”, PhD thesis, Harbin Institute Of Technology, 2009.

[2] Chang Xuenian, and Yao Yi, "The development and investigation of displacement sensor”, Metrology \&Measurement Technique, vol. 36, pp. 6-11, 2009.

[3] D. Francis, D. Masiyano, J. Hodgkinson and R. Tatam, (). A mechanically stable laser diode speckle interferometer for surface contouring and displacement measurement. Meas. Sci. Technol., vol. 26, 055402. 2015.

[4] Zhang Jianqing, Pan Li and Wang Shugen, photogrammetry, Wuhan University Press, 2003.

[5] A.Stumpf, J. Malet, P. Allemand, M. Pierrot-Deseilligny and Skupinski, G. Ground-based multi-view photogrammetry for the monitoring of landslide deformation and erosion. Geomorphology, vol. 17, pp 130-145, 2015.

[6] Xiong Zhang, Lin Li and Gang Chen. A photogrammetry-based method to measure total and local volume changes of unsaturated soils during triaxial testing. Acta Geotechnica, vol. 10, pp. 55-82, 2015

[7] Li Xiujuan, Zhou Yan and Xiong Hanjiang. 3d indoor location on mobile phones using embedded sensors and close-range photogrammetry. Lecture Notes in Geoinformation \& Cartography, pp79-90, 2015.

[8] Chang Xuenian, and Yao Yi, "The development and investigation of displacement sensor”, Metrology \&Measurement Technique, vol. 36, pp. 42-44, 2009.

[9] Zhou Guanjie, and Zhao Yujie, "Characteristics of structure design for spaceborne radar”, Electro-Mechanical Engineering, vol. 24, pp. 35-40, 2008.

[10] Guan Hongshan, "Study on thermal control design of satelliteborne SAR antenna”, Radar Science and Technology, vol. 5, pp. 427-430, 2007.

[11] Yu Ying, "Research on key technologies of binocular stereo industrial photogrammetry and its applications”, PhD thesis, PLA Information Engineering University, 2010. 